\title{
Influence of stimulus symmetry and complexity upon haptic scanning strategies during detection, learning, and recognition tasks
}

\author{
PAUL J. LOCHER \\ Montclair State College, Upper Montclair, New Jersey 07043 \\ and \\ ROGER W. SIMMONS \\ San Diego State University, San Diego, California 92115
}

\begin{abstract}
Two experiments were conducted to investigate the perceptual processes involved in haptic exploration of randomly generated shapes. Experiment 1 required subjects to detect the symmetrical or asymmetrical characteristirs of individually presented plastic shapes. The shapes also varied in complexity as measured by the number of sides. Experiment 2 involved learning a set of shapes and then attempting to recognize these "old" shapes when presented together with a series of "new" shapes. In both experiments, reaction time was investigated as a function of stimulus complexity and task requirements. Furthermore, it was observed that an initial orienting response was used to direct the subjects to adopt specific scanning strategies when examining asymmetrical or symmetrical shapes. The influences of stimulus properties and task requirements upon haptic encoding processes are discussed.
\end{abstract}

The first stage in the processing of sensory information involves the active exploration of the stimulus field during which portions of the field are selected. For the visual mode, the study of eye movements has made important contributions to the understanding of the relationship between the informational content and structure of a visual field, its perceived complexity, a subject's exploratory activity, and the encoding processes and higher mental processing operations (Monty \& Senders, 1976). For example, the form parameter of symmetry has been examined extensively with respect to its influence on subjective evaluations of stimulus complexity, visual scanning strategies, and detection and recognition performance involving a wide variety of stimuli (e.g., Carmody, Nodine, \& Locher, in press; Corballis \& Roldan, 1975; Day, 1968; Locher $\&$ Nodine, 1973). With the exception of the work of Davidson (Davidson, 1972; Davidson, Abbott, \& Gershenfeld, 1974; Davidson \& Whitson, 1974), research in the field of haptic perception has contributed little to an understanding of the relationship

Portions of this paper were presented at the annual conference of the North American Society for the Psychology of Sport and Physical Activity, Ithaca, New York, May 24, 1977. We thank Ms. Pat Kaylor for running subjects. Requests for reprints should be sent to Paul Locher, Department of Psychology, Montclair State College, Upper Montclair, New Jersey 07043. between configurational properties of form, haptic scanning strategies, and performance on learning, discrimination, and recognition tasks.

Davidson (1972) has demonstrated that there is an interaction between stimulus properties, haptic scanning strategies, and haptic judgments of curvature by blind and sighted subjects. In addition, Davidson and Whitson (1974) found that the accuracy in judgments on a haptic curvature matching task was determined, in part, by an interaction between task requirements and search strategies. These findings emphasize the need for a thorough investigation of the psychophysics of form for the haptic mode and the interaction effect of form parameters upon haptic encoding processes and performance on perceptual tasks.

Frequently, stimuli used in haptic perception studies are simple and they lack variation on even a single form parameter. Landrigan and Forsyth (1974) presented subjects with only 12-sided forms, Butter and Bjorklund (1976) used only 10-sided random forms as stimuli, and the often used Gibson free-form bodies (Davidson, Abbott, \& Gershenfeld, 1974; Davidson, Barnes \& Mullen, 1974) consist of only five form protuberances. Those studies (Millar, 1974; Zung, Butter, \& Cashdan, 1974) which have used forms differing on a number of dimensions (size, number of turns, length of angles, etc.) have not examined the influence of these aspects of 
structural complexity upon subjects' performance. In addition, with the exception of the aforementioned work of Davidson, none of the above studies have examined haptic scanning strategies and, as Davidson and Whitson have pointed out, "little is known of the role of such movements in perception" (p. 687).

According to Neisser (1976), "we do not have adequate terms to describe the types of information that are available to the touch nor the types of movements that the observer must make to obtain them" (p. 26). The purpose of the present study was to examine the role of the form parameters of symmetry and complexity as measured by the number of sides (turns) in the perimeter of a shape upon haptic scanning processes and upon subjects' ability to detect symmetry. In a second experiment, the influence of the same two form parameters was examined with respect to haptic scanning processes and performance on a learning and recognition task.

\section{EXPERIMENT 1}

\section{Method}

Subjects. Twelve right-hand-dominant undergraduate students (six males and six females) volunteered to serve as subjects.

Stimuli. Method 4 of Attneave and Arnoult (1956) was 'used to generate the shapes presented in Figure 1. The shapes differed on two form parameters: symmetry (symmetrical or asymmetrical) and complexity as measured by the number of sides (turns) in the perimeter of the shape $(12,18,24$, or 30 sides). This combination of variables resulted in a set of eight shapes. Three different sets of shapes were generated, yielding a total of 24 stimuli. The shapes, which were originally prepared on graph paper, were reproduced in 1/8-in. pieces of plastic. The shapes measured approximately $15 \mathrm{~cm}$ wide $\times 19 \mathrm{~cm}$ long.

Apparatus. The stimuli were displayed individually on a pedestal raised $10 \mathrm{~cm}$ above the base of the display unit. Stimuli lay in a horizontal plane; the axis of symmetry for symmetrical shapes lay in the sagittal plane of the subject. Two $1 / 8$-in. holes were drilled through each shape, which facilitated mounting of the shapes onto two dowls that extended from the pedestal. The ends of the dowls were flush with the top surface of the shapes and in no way interfered with the scanning procedures used by the subjects. A curtain drawn across the front of the display unit allowed free movement of the hand over the stimulus and simultaneously excluded the use of vision.

The subject's exploratory activity was monitored and taped using an $\mathrm{ABC}$ video camera placed approximately $1.5 \mathrm{~m}$ above and in line with the longitudinal axis of the stimulus. This position of the camera gave a clear view of finger and wrist movements. Each subject's exploratory activity and verbal responses were continuously recorded on videotape through the use of a SONY 3650 videotape recorder.

Procedure. Before testing commenced, each subject was required to visually identify a series of symmetrical and asymmetrical shapes (not included in the set of stimuli) illustrated on a card to ensure that the subject understood the concept of shape symmetry.

The subject was seated in front of the display unit such that the right shoulder was in line with the longitudinal axis of the stimulus. The experimenter placed the subject's middle finger of the right hand on a small indentation located in the center of the shapes. The subject was instructed to hold the other fingers in

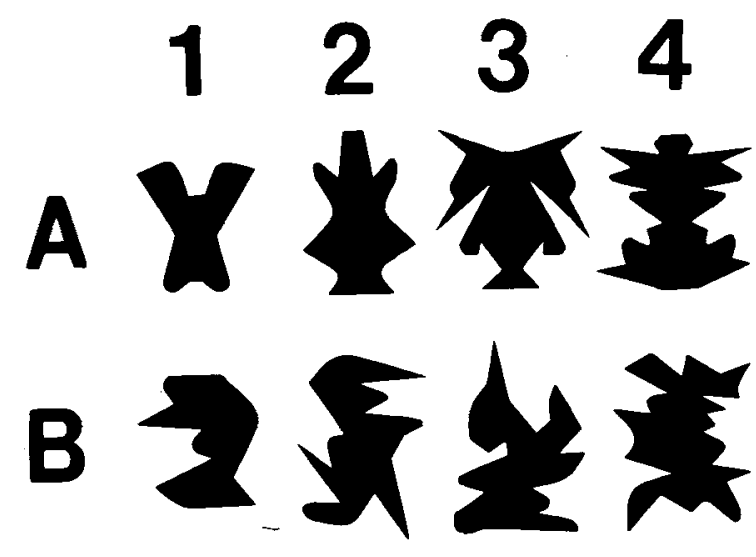

Figure 1. Eight nonrepresentational shapes used in the experiment Columns 1, 2, 3, and 4 consist of, respectively, 12-, 18-, 24-, and 30-sided shapes. Rows $A$ and $B$ consist of symmetrical and asymmetrical shapes, respectively.

an outstretched position above the stimulus. With the command "begin," the subject was free to use all fingers to determine the symmetry of the shape. His task was to respond as quickly and accurately as possible to the symmetry of the shape and to respond accordingly, either "symmetrical" or "asymmetrical." Having responded, the subject removed his hand to a position above the pedestal and awaited the presentation of the next stimulus. The subject was taken through a series of practice trials using both symmetrical and asymmetrical shapes not included in the set of stimuli until the experimenter was convinced that the subject understood the task.

The testing session was split into two blocks of 24 trials each. Each trial consisted of the presentation of a single stimulus, the order of presentation being randomized for each subject. After a 5-min rest interval, during which the set of stimuli were again randomized and then rotated $180^{\circ}$ about the longitudinal axis, the second block of trials was begun, following the identical procedure of the first block. This resulted in each subject scanning each of 24 stimuli twice.

Videotape scoring. The taped record for each subject was independently analyzed twice by two scorers. On the first viewing, the elapsed time in seconds from the command "begin" to the subject's verbal response was recorded for each stimulus. Interrater reliability on this task was high $(r=.95)$. The scanning strategy used by each subject to examine each stimulus was independently assessed by the two scorers during a second viewing of the videotape. These strategies are described below. In those situations in which reaction time for a stimulus was very fast ( $4 \mathrm{sec}$ or less), the tape was played at slow motion speed to improve accuracy in identifying the scanning strategy.

Scanning strategies. A pilot study resulted in the categorization of exploratory activity into six scanning strategies. Three methods of scanning were defined as follows. A simultaneous apprehension scan consisted of smooth, continuous movements of the thumb and one other finger, usually the middle finger, over stimulus features directly across from each other along the longitudinal axis of the shape. For symmetrical shapes, mirrorimage features were continuously and simultaneously scanned using this strategy. A trace scan consisted of the thumb, index and middle fingers, held together, sliding smoothly and continuously along one edge of the shape. Occasionally, one or more fingers were curled over the edge of the shape or they were used in a pincer fashion to carefully examine a single feature of the shape. Features along one-third of one side of the shape were examined, followed by a similar scan on the opposite side of the 
shape to check for the presence of symmetrical counterparts. The use of both simultaneous apprehension and trace strategies to scan a single shape was defined as being a mixed strategy.

In addition to the three methods of scanning, two search processes were defined as complete scan-involving one or more complete sweeps of the shape-and partial scan-involving less than one full sweep of the shape. Thus, the scanning strategy used by each subject on each shape was classified as to the method of scanning and the search process (e.g., simultaneous apprehension/complete, trace/partial, etc.).

\section{Results}

Error data. Subjects made an average of 3.5 $(\mathrm{SD}=2.4)$ incorrect identifications of the 48 stimuli. Of the responses made to symmetrical shapes, 10 subjects made one or more incorrect identifications, resulting in a total of $11.8 \%$ incorrect responses. Three subjects were responsible for the $2.6 \%$ incorrect identifications of asymmetrical shapes.

Reaction time data. A 2 (symmetry) by 4 (com: plexity) by 2 (blocks) randomized block factorial design with repeated measures (Kirk, 1968) was used to examine the reaction time data presented in Table 1. The dependent variable was the mean reaction time in seconds per subject for the three stimuli in each of the eight combinations of shape parameters per block of trials.

A significant main effect for symmetry was found, $F(1,11)=10.16, p<.01$. Subjects spent more time examining symmetrical shapes $(9.55 \mathrm{sec})$ than asym- metrical shapes $(7.38 \mathrm{sec})$. In addition, reaction time varied significantly with shape complexity, $F(3,33)$ $=6.18, \mathrm{p}<.005$. More time was spent examining 30 -sided shapes than 12 -sided shapes $(p<.01$, Tukey test) or 18 -sided shapes $(p<.05$, Tukey test). No significant main effect for blocks of trials was obtained, $\mathrm{F}(1,11)=.06$, and it was concluded that a practice effect did not occur between Blocks 1 and 2 . None of the interactions was significant.

Scanning strategies. Table 2 presents the frequency of occurrence of the six scanning strategies described in the Methods section for each treatment level. For symmetrical shapes, significantly more complete strategies $(83 \%)$ were used in identifying symmetry than partial strategies $(17 \%), \chi^{2}(1)=79.34, p<.001$. Of the complete strategies, approximately the same number were of the simultaneous apprehension or mixed type with significantly fewer trace strategies being observed, $\chi^{2}(2)=40.35, p<.001$. No significant difference in the type of partial scan strategies used to identify symmetrical shapes was found, $\chi^{2}$ $=1.29$.

For asymmetrical shapes, significantly more partial scan strategies $(63 \%)$ were observed than complete scan strategies $(37 \%), \chi^{2}(1)=20.54$, $\mathrm{p}<.001$. These partial scan strategies were predominately trace as compared to either simultaneous apprehension or mixed strategies, $\chi^{2}(2)=72.97$, $\mathrm{p}<.001$. An uneven distribution of complete scan strategies for asymmetrical shapes was observed in

Table 1

Mean Reaction Time in Seconds for Each Treatment Level

\begin{tabular}{|c|c|c|c|c|c|c|c|c|c|c|}
\hline \multirow[b]{3}{*}{ Symmetry } & \multirow{3}{*}{$\begin{array}{c}\text { Blocks of } \\
\text { Trials }\end{array}$} & \multicolumn{9}{|c|}{ Complexity } \\
\hline & & \multicolumn{2}{|c|}{12} & \multicolumn{2}{|c|}{18} & \multicolumn{2}{|c|}{24} & \multicolumn{2}{|c|}{30} & \multirow{2}{*}{$\begin{array}{l}\text { Row } \\
\text { Mean }\end{array}$} \\
\hline & & Mean & SD & Mean & SD & Mean & SD & Mean & SD & \\
\hline Symmetrical & $\begin{array}{l}\text { First } \\
\text { Second }\end{array}$ & $\begin{array}{l}8.46 \\
9.31\end{array}$ & $\begin{array}{l}3.66 \\
4.09\end{array}$ & $\begin{array}{l}9.09 \\
8.90\end{array}$ & $\begin{array}{l}3.46 \\
2.56\end{array}$ & $\begin{array}{r}9.58 \\
11.15\end{array}$ & $\begin{array}{l}3.35 \\
4.48\end{array}$ & $\begin{array}{l}10.08 \\
10.11\end{array}$ & $\begin{array}{l}4.22 \\
3.18\end{array}$ & $\begin{array}{l}9.30 \\
9.80\end{array}$ \\
\hline Asymmetrical & $\begin{array}{l}\text { First } \\
\text { Second }\end{array}$ & $\begin{array}{l}6.68 \\
6.32\end{array}$ & $\begin{array}{l}3.30 \\
2.33\end{array}$ & $\begin{array}{l}7.13 \\
6.51\end{array}$ & $\begin{array}{l}2.83 \\
1.92\end{array}$ & $\begin{array}{l}8.13 \\
7.33\end{array}$ & $\begin{array}{l}3.64 \\
2.84\end{array}$ & $\begin{array}{l}9.18 \\
7.66\end{array}$ & $\begin{array}{l}4.49 \\
2.77\end{array}$ & $\begin{array}{l}7.78 \\
6.98\end{array}$ \\
\hline Column Mean & & 7.69 & & 7.94 & & 8.83 & & 9.40 & & \\
\hline
\end{tabular}

Table 2

Frequency of Haptic Scanning Strategies for Each Treatment Level

\begin{tabular}{|c|c|c|c|c|c|c|c|c|c|c|c|c|c|}
\hline \multicolumn{2}{|r|}{ Process } & \multicolumn{6}{|c|}{$\begin{array}{c}\text { Symmetrical Shapes } \\
\text { Complexity }\end{array}$} & \multicolumn{6}{|c|}{$\begin{array}{c}\text { Asymmetrical Shapes } \\
\text { Complexity }\end{array}$} \\
\hline Search & Scanning & 12 & 18 & 24 & 30 & Total & $\% *$ & 12 & 18 & 24 & 30 & Total & $\% *$ \\
\hline Complete & $\begin{array}{l}\text { Simultaneous Apprehension } \\
\text { Trace } \\
\text { Mixed }\end{array}$ & $\begin{array}{r}34 \\
4 \\
26\end{array}$ & $\begin{array}{l}26 \\
12 \\
24\end{array}$ & $\begin{array}{r}23 \\
9 \\
26\end{array}$ & $\begin{array}{r}19 \\
8 \\
28\end{array}$ & $\begin{array}{r}102 \\
33 \\
104\end{array}$ & $\begin{array}{l}35 \\
12 \\
36\end{array}$ & $\begin{array}{r}4 \\
9 \\
13\end{array}$ & $\begin{array}{r}6 \\
6 \\
17\end{array}$ & $\begin{array}{r}5 \\
10 \\
14\end{array}$ & $\begin{array}{r}2 \\
6 \\
14\end{array}$ & $\begin{array}{l}17 \\
31 \\
58\end{array}$ & $\begin{array}{r}6 \\
11 \\
20\end{array}$ \\
\hline Partial & $\begin{array}{l}\text { Simultaneous Apprehension } \\
\text { Trace } \\
\text { Mixed }\end{array}$ & $\begin{array}{l}5 \\
0 \\
3\end{array}$ & $\begin{array}{l}4 \\
3 \\
3\end{array}$ & $\begin{array}{l}4 \\
5 \\
5\end{array}$ & $\begin{array}{l}5 \\
5 \\
7\end{array}$ & $\begin{array}{l}18 \\
13 \\
18\end{array}$ & $\begin{array}{l}6 \\
5 \\
6\end{array}$ & $\begin{array}{r}11 \\
27 \\
8\end{array}$ & $\begin{array}{r}6 \\
23 \\
14 \\
\end{array}$ & $\begin{array}{r}3 \\
28 \\
12 \\
\end{array}$ & $\begin{array}{r}6 \\
36 \\
8 \\
\end{array}$ & $\begin{array}{r}26 \\
114 \\
42 \\
\end{array}$ & $\begin{array}{r}9 \\
40 \\
14 \\
\end{array}$ \\
\hline
\end{tabular}

*Based upon a total of 288 responses (12 subjects $\times 24$ stimuli). 
the data, $\chi^{2}(2)=24.60, \mathrm{p}<.001$, with mixed strategy being the most frequently used of this group.

When the distribution of strategies by subject was examined, the data indicated that, with the exception of two subjects, the frequency of strategy usage on an individual basis for each treatment level was similar to that of the grouped data. Of the two exceptions, one subject used complete scan strategies exclusively to identify stimuli and a second subject used 43 complete scan strategies to identify the total set of 48 stimuli.

It can be seen from Table 2 that the type of scanning strategy used by subjects to detect symmetry was not influenced by the complexity of the shape.

\section{Discussion}

For both symmetrical and asymmetrical shapes, it was hypothesized that the detection of symmetry would require the examination of a few shape features (partial scan) rather than an exhaustive or complete search of the shape which would be required to memorize or "learn" a shape. The procedure used to ensure that subjects understood the concept of shape symmetry and the instructions to the subjects emphasized the fact that shapes were either totally symmetrical or asymmetrical. It was hypothesized, therefore, that partial information from either type of stimulus should have been sufficient to make a decision concerning the symmetry of a shape. The data did not support this hypothesis. A partial/trace scanning strategy was predominately used to examine asymmetrical shapes. However, subjects overwhelmingly used complete scan strategies of either the simultaneous apprehension or mixed type to examine symmetrical shapes. This difference in strategies resulted in more time being spent scanning symmetrical shapes than asymmetrical shapes. These findings, along with the observed difference in error rate, suggest that symmetrical shapes are more difficult to detect haptically than asymmetrical shapes.

Differences in the type of haptic scanning strategies used to examine symmetrical and asymmetrical shapes were observed in the data. Stronger evidence of this difference is described in Experiment 2 . How did a subject initially decide to use a trace strategy for asymmetrical shapes and a simultaneous apprehension or mixed strategy for symmetrical shapes? It was observed from the videotape records that once given the command to begin, initial contact with the shape was made in one of two ways by all subjects. Either the four fingers moved back and forth across the top of the shape in a large generalized movement or, from the starting position, the fingers were placed over the top edge of the shape and held there momentarily. It is hypothesized that either of these movements provided input which made possible a tentative decision concerning the symmetry of the shape and resulted in an orienting response similar in function to the global response described by Carmody, Nodine, and Locher (in press) for the visual system. This cursory information concerning the symmetry of the shape directs the subject to use a strategy which he thinks will serve to verify the orienting response.

For asymmetrical shapes, only a small quantity of additional input (scanning of approximately one-third of the shape's edge) was necessary to substantiate the orienting response, as indicated by the partial scan strategies which characterized the scanning of asymmetrical shapes. Similarly, one would hypothesize that the use of simultaneous apprehension strategies (or mixed strategies) to examine shapes believed to be symmetrical should facilitate the speed of their detection. A simultaneous apprehension strategy as defined above should enable the perceiver to quickly detect, through simultaneous input from symmetrical features of the shape, the mirror-image quality of symmetrical shapes (Davidson \& Whitson, 1974; Lappin \& Foulke, 1973). The extensive use of complete scan strategies to examine symmetrical shapes and the greater scanning time at all levels of complexity for symmetrical shapes as compared to asymmetrical shapes demonstrate that the above hypothesis is false. Simultaneous apprehension strategies did not facilitate the speed of detection of symmetrical shapes.

Two possible explanations of the lack of support for this hypothesis are suggested in the literature. Butter and Bjorklund (1976) found that neither $5 \mathrm{sec}$ nor $10 \mathrm{sec}$ of one-handed haptic exploration of 10-sided random forms yielded more usable information, as measured by the accuracy of reproduction of the form, than did two-handed exploration for comparable periods of time. They suggest that this lack of efficiency of a "simultaneous scan" strategy may be due to the fact that subjects have not learned to use more efficient search strategies. In support of this contention, Davidson (1972) has established that directing subjects to use a particular kind of haptic scanning technique improves judgments of curvature made with one hand. Furthermore, Davidson and Whitson (1974) suggest that scanning differences and the greater accuracy of blind subjects compared to sighted subjects on a haptic curvature matching task may be the result of perceptual experience.

Based upon the limitations of the haptic sensory system (Neisser, 1976), an alternate view suggests that haptic perception is restricted to a serial process of exploration (trace scan) and information pickup when one hand is used (Butter \& Bjorklund, 1976). 
As such, haptic information must be encoded serially to allow the subject to focus attention on an informative stimulus feature, to reduce interference among multiple inputs from several fingers, and to facilitate the veridical integration of stimulus features. Increased scanning time as a function of structural complexity for both symmetrical and asymmetrical shapes as observed in the present study lends support to the view that haptic perception demands serial encoding processes. Furthermore, this finding is consistent with the prediction of serial models that reaction time increases with the number of relevant dimensions (complexity) of stimuli (e.g., Egeth, 1966).

\section{EXPERIMENT 2}

\section{Method}

Subjects. Seven right-hand-dominant undergraduate students (four females and three males) volunteered to serve as subjects. None of these subjects participated in Experiment 1.

Procedure. The stimuli, apparatus, video recording procedures, and videotape analysis procedures of Experiment 1 were used in Experiment 2.

The subject was seated in front of the apparatus, and the experimental procedure, which consisted of a learning phase and a recognition phase, was explained to the subject. During the learning phase, the subject was presented with four shapes representing each level of complexity and both levels of symmetry (e.g., 12-sided symmetrical; 18-sided asymmetrical; 24-sided symmetrical, and 30-sided asymmetrical). The subject was allowed to scan each stimulus for as long as was necessary to "remember" the shape. He was informed that he would be asked to recognize the shape at a later point in the experiment. Several practice trials utilizing shapes not included in the set of stimuli preceded the learning phase in order to acquaint the subject with the apparatus and procedure.

The eight stimuli included in the recognition phase consisted of a random arrangement of the four learning phase shapes ("old") and four new shapes. The four new shapes represented each level of complexity and both levels of symmetry. The subject was given unlimited time to scan each shape and respond "old" if he recognized the shape as one from the learning phase and "new" if he had not experienced the shape before.

The entire experimental procedure was repeated for each subject after a 5-min rest period utilizing a new set of stimulus shapes. This resulted in the presentation of a total of eight shapes, including all levels of form parameters during both learning phases. These eight shapes constituted the "old"' shapes of both recogni- tion phases. A second set of eight shapes, including all levels of form parameters, comprised the "new" shapes for both recognition phases.

The experimental procedure lasted approximately $20 \mathrm{~min}$ for each subject. The selection of old and new shapes for each subject was completely randomized.

\section{Results}

Error data. The error rate for the combined recognition phases was $26.8 \%$. Errors were distributed evenly between symmetrical shapes $(53 \%)$ and asymmetrical shapes $(47 \%)$ ). Subjects made an average of 4.3 errors $(\mathrm{SD}=2.9)$ to the 16 stimuli constituting both recognition phases.

Reaction time data. The reaction time data presented in Table 3 was analyzed through the use of a 2 (symmetry) by 4 (complexity) by 3 (experience with shape) randomized block factorial design (Kirk, 1968). The three levels of experience with shape were (1) learning phase, (2) experience with "old" shapes during the recognition phase, and (3) experience with "new" shapes during recognition. The dependent variable was reaction time in seconds. $\log _{10}$ transformations of the data were necessary to achieve the symmetry of the variance-covariance matrix required for the use of analysis of variance with repeated measures.

A significant main effect for complexity was found, $F(3,18)=3.94, p<.05$, indicating that reaction time increased with complexity. None of the interactions involving this variable were found to be significant. Subjects spent more time examining 24 and 30-sided shapes than 12 -sided shapes $(p<.05$, Tukey test). All other differences between means were not significant.

Although no significant main effect for symmetry was found, $F(1,6)=2.19$, the presence of a significant main effect for the variable experience with shape, $F(2,12)=11.37, p<.005$, together with a significant Symmetry by Experience with Shape interaction, $F(2,12)=6.39, p<.025$, led to a simple main effects analysis on the variable of symmetry. For asymmetrical shapes, the reaction times for the three levels of experience with shape were significant-

Table 3

Mean Reaction Time in Seconds for Each Treatment Level

\begin{tabular}{|c|c|c|c|c|c|c|c|c|c|}
\hline \multirow[b]{3}{*}{ Task } & \multirow[b]{3}{*}{ Symmetry } & \multicolumn{8}{|c|}{ Complexity } \\
\hline & & \multicolumn{2}{|c|}{12} & \multicolumn{2}{|c|}{18} & \multicolumn{2}{|c|}{24} & \multicolumn{2}{|c|}{30} \\
\hline & & Mean & SD & Mean & SD & Mean & SD & Mean & SD \\
\hline Learning Phase & $\begin{array}{l}\text { Symmetrical } \\
\text { Asymmetrical }\end{array}$ & $\begin{array}{l}31.9 \\
45.4\end{array}$ & $\begin{array}{l}19.6 \\
23.9\end{array}$ & $\begin{array}{l}36.3 \\
51.1\end{array}$ & $\begin{array}{r}9.6 \\
22.8\end{array}$ & $\begin{array}{l}51.7 \\
56.7\end{array}$ & $\begin{array}{l}24.8 \\
28.2\end{array}$ & $\begin{array}{l}55.1 \\
58.2\end{array}$ & $\begin{array}{l}24.7 \\
25.0\end{array}$ \\
\hline $\begin{array}{l}\text { Recognition of } \\
\text { "Old" Shapes }\end{array}$ & $\begin{array}{l}\text { Symmetrical } \\
\text { Asymmetrical }\end{array}$ & $\begin{array}{l}28.7 \\
31.4\end{array}$ & $\begin{array}{l}25.6 \\
21.2\end{array}$ & $\begin{array}{l}23.2 \\
40.8\end{array}$ & $\begin{array}{l}24.9 \\
19.4\end{array}$ & $\begin{array}{l}27.5 \\
37.1\end{array}$ & $\begin{array}{l}20.8 \\
14.3\end{array}$ & $\begin{array}{l}38.7 \\
39.7\end{array}$ & $\begin{array}{l}36.5 \\
25.2\end{array}$ \\
\hline $\begin{array}{l}\text { Examination of } \\
\text { "New" Shapes }\end{array}$ & $\begin{array}{l}\text { Symmetrical } \\
\text { Asymmetrical }\end{array}$ & $\begin{array}{l}34.7 \\
24.2\end{array}$ & $\begin{array}{l}25.5 \\
14.3\end{array}$ & $\begin{array}{l}35.1 \\
30.0\end{array}$ & $\begin{array}{l}17.9 \\
17.8\end{array}$ & $\begin{array}{l}39.5 \\
34.1\end{array}$ & $\begin{array}{l}20.1 \\
14.5\end{array}$ & $\begin{array}{l}37.3 \\
27.4\end{array}$ & $\begin{array}{l}27.5 \\
17.1\end{array}$ \\
\hline
\end{tabular}


ly different, $F(2,12)=12.46, p<.005$. Specifically, subjects spent more time examining each shape during the learning phase ( $53.0 \mathrm{sec}$ per shape) than in the recognition of "old" shapes ( $37.4 \mathrm{sec}$ per shape) or examination of "new" shapes (29.1 sec per shape) $(\mathrm{p}<.01$, Tukey test). However, the difference in reaction time for "old" and "new" shapes during recognition was not significant.

A significant simple main effect for the variable experience with shape was found for symmetrical shapes, $F(2,12)=6.74, p<.025$. On the average, subjects spent $44.1 \mathrm{sec}$ examining each symmetrical shape during the learning phase. This was found to be a significantly greater amount of time $(p<.05$, Tukey test) than was required for recognition of "old" shapes (28.4 sec per shapes), but not greater than the $38.9 \mathrm{sec}$ taken by subjects to examine each "new" shape.

Scanning strategies. Subjects used trace strategies almost exclusively (95\%) during both phases of Experiment 2 to examine asymmetrical shapes. The remaining $5 \%$ of the strategies were mixed; not a single simultaneous apprehension scan was observed in the data for asymmetrical shapes. For symmetrical shapes, the frequencies of trace, simultaneous apprehension, and mixed scanning strategies for the learning phase were $46 \%, 18 \%$, and $36 \%$, respectively; for recognition of "old" shapes, the values were $50 \%, 25 \%$, and $25 \%$, and for the examination of "new" shapes, $57 \%, 18 \%$, and $25 \%$.

Since all subjects used complete scan search processes in both phases of the experiment, the number of times the hand crossed the midline of the shape (sweep) was recorded and the data subjected to the same analysis as that used for the reaction time data. The results of this analysis were comparable to that of the reaction time data with respect to all main effects and interactions; that is, the greater the reaction time, the greater the number of sweeps the subject made to the shape. For example, the reaction times for the three levels of experience with shape-learning phase, experience with "old" shapes, and experience with "new" shapes-were $48.3,32.8$, and $32.9 \mathrm{sec}$, respectively, and 4.8, 3.7, and 3.7 swceps, respectively.

\section{Discussion}

In contrast to the detection of symmetry which can be accomplished, at least for asymmetrical shapes, using partial information from the stimulus, a learning task necessitates that the subject scan the entire shape at least once to find and to encode distinctive features of that shape. This, in fact, was found to be the case for both types of symmetry and for both the learning and recognition task. In addition to the use of a complete scan strategy, learning and recognition tasks necessitate the sequential input of stimulus fea- tures. If haptic exploration demands serial processing (Butter \& Bjorklund, 1976), then for asymmetrical shapes, the use of a simultaneous apprehension strategy would result in the inability to focus attention on individual features due to multiple inputs arising from the simultaneous encoding of asymmetrical features. In addition, multiple input from an asymmetrical shape would interfere with the veridical integration of successive inputs in memory. Therefore, a strategy which would provide single, sequential input of stimulus features would be expected. This was the case; $95 \%$ of all strategies used for both learning and recognition of asymmetrical shapes were trace scans.

The almost exclusive use of trace scans to examine asymmetrical shapes supports the hypothesis that an orienting response directs subjects to use appropriate encoding strategies. Verification of the existence of an orienting response for the haptic mode and its influence upon a decision concerning the strategy to be used needs careful examination.

Learning and recognition tasks involving symmetrical shapes, as well as detection of symmetrical shapes, should be facilitated by the simultaneous encoding of mirror image features. Such strategies should result in the reduction of information, and possibly facilitate the veridical organization in memory of the stimulus (Davidson, 1972). The data did not support this prediction. Eighteen percent of the scanning strategies used during the learning task were of the simultaneous apprehension type and $21 \%$ of the strategies used during recognition were simultaneous apprehension scans. For both tasks, the dominant strategy was a trace scan.

This discrepancy between the strategy used by subjects and the hypothesized strategy may be due to the lack of experience of subjects with haptic search tasks, as pointed out in the previous Discussion section. Directing subjects to use a simultaneous apprehension scan to encode and recognize symmetrical shapes may result in a decrease in reaction time and be accompanied by a comparable decrease in sweeps. The data provide evidence that the use of simultaneous apprehension and mixed strategies to encode symmetrical shapes may facilitate the processing of stimulus feature information. The use of simultaneous apprehension $(18 \%)$ and mixed $(36 \%)$ strategies by subjects during the learning phase was probably responsible for the fact that reaction time for symmetrical shapes was significantly less than that for asymmetrical shapes. This faster reaction time for symmetrical shapes did not, however, result in an increased error rate. Subjects made as few errors in recognizing symmetrical shapes (17) as in recognizing asymmetrical shapes (14).

The effects of task requirements upon the encoding processes for symmetrical and asymmetrical 
shapes are further indicated by the reaction time data for Experiment 2. Encoding symmetrical shapes required less time than for asymmetrical shapes. However, detection of asymmetrical shapes (Experiment 1) was faster than for symmetrical shapes. In addition, subjects required less time to recognize symmetrical shapes than asymmetrical shapes. These findings indicate that it is easier to encode a symmetrical stimulus and that a decision concerning the presence of symmetrical shapes stored in memory can be made more quickly than for asymmetrical shapes.

In summary, our data indicate that there is an interaction effect of stimulus properties and task requirements upon haptic scanning processes. Much additional investigation of the psychophysics of form for the haptic mode and the effect of form parameters upon haptic encoding processes and performance on perceptual tasks is needed.

\section{REFERENCES}

Attneave, F., \& Arnoult, M. D. The quantitative study of shape and pattern perception. Psychological Bulletin, 1956, 53, 452-471.

Butter, E. J., \& Bjorklund, D. F. Are two hands better than one? Assessing information acquired from one- and two-handed haptic exploration of random forms. Perceptual and Motor Skills, 1976, 43, 115-120.

Carmody, D., Nodine, C., \& Locher, P. The global detection of symmetry. Perceptual and Motor Skills, in press.

Corballis, M. C., \& Roldan, C. E. Detection of symmetry as a function of angular orientation. Journal of Experimental Psychology: Human Perception and Performance, 1975, 1, 221-230.
Davidson, P. W. Haptic judgments of curvature by blind and sighted humans. Journal of Experimental Psychology, 1972, 93, 43-55.

Davidson, P. W., Aвbotr, S., \& Gershenfeld, J. Influence of exploration time on haptic and visual matching of complex shapes. Perception \& Psychophysics, 1974, 15, 539-543.

Davidson, P. W., Barnes, J., \& Mullen, G. Differential effects of task memory demand on haptic matching of shape by blind and sighted humans. Neuropsychologia, 1974, 12, 395-397.

Davidson, P. W., \& Whitson, T. T. Haptic equivalence matching of curvature by blind and sighted humans. Journal of Experimental Psychology, 1974, 102, 687-690.

DAY, $H$. The importance of symmetry and complexity in the evaluation of complexity, interest, and pleasingness. Psychonomic Science, 1968, 10, 339-340.

Egeth, H. E. Parallel versus serial processes in multidimensional stimulus discrimination. Perception \& Psychophysics, 1966, 1 , 245-252.

KIRK, R. E. Experimental design: Procedures for the behavioral sciences. Belmont, Calif: Brooks/Cole, 1968.

Landrigan, D. T., \& Forsyth, G. A. Regulation and reproduction of movement effects in exploration-recognition performance. Journal of Experimental Psychology, 1974, 103, 1124-1130.

LAPPIN, J. S., \& FoulkE, E. Expanding the tactual field of view. Perception \& Psychophysics, 1973, 14, 237-241.

LOCHER, P. J., \& Nodine, C. F. Influence of stimulus symmetry on visual scanning patterns. Perception \& Psychophysics, 1973, 13, 408-412.

MillaR, S. Tactile short-term memory by blind and sighted children. British Journal of Psychology, 1974, 65, 253-262.

Monty, R. A., \& SENDERs, J. W. Eye movements and psychological processes. New York: Wiley, 1976.

NEISSER, U. Cognition and reality. San Francisco: Freeman, 1976.

ZunG, B. J., Butter, E. J., \& Cashdan, S. Visual-haptic form recognition with task delay and sequenced bimodal input. Neuropsychologia, 1974, 12, 73-81.

(Received for publication April 25, 1977; revision accepted November $22,1977$. ) 